\title{
Infection prevention and control (IPC) at a Lassa fever treatment center before and after the implementation of an intensive IPC program
}

\author{
Olayinka Stephen Ilesanmi ${ }^{*}$, Oladele Olufemi Ayodeji ${ }^{2}$, Ayobami A. Bakare ${ }^{3}$, Nelson Adedosu ${ }^{4}$, \\ Anthonia Adeagbo ${ }^{5}$, Adedamola Odutayo ${ }^{6}$, Felix Olugbenga Ayun ${ }^{6}$, Ayomide E. Bello ${ }^{7}$
}

\begin{abstract}
Background: Infection prevention and control (IPC) programs are important to control the Lassa Fever (LF) outbreak. We reported IPC's status at the Federal Medical Centre, Owo, southwest Nigeria, before and after implementing the IPC program during a surge in the LF outbreak.

Methods: We conducted a longitudinal observational study among five health care professionals at the Federal Medical Centre, Owo, between February 2019 and May 2019 using the IPC Assessment Framework (IPCAF). The tool has eight core components with a score of 0-100 per component and provided a baseline assessment of the IPC program and evaluation after three months. We interviewed relevant unit heads and IPC committee members in the first phase. In the second phase, we designed and implemented the IPC program, and in the third phase, we conducted a repeat interview similar to the first phase. The program initiated included training healthcare workers and providing relevant IPC items according to identified gaps and available funding.

Results: We interviewed five health care professionals, two female nurses, and three male doctors responsible for organizing and implementing IPC activities at the Federal Medical Centre, Owo, with an in-depth understanding of IPC activities. The overall IPC level score increased from 318.5 at baseline to 545 at three months later. IPC improvements were reported in all the components, with IPC education and training [baseline (20), final (70)], IPC guidelines [baseline (50), final (92.5)] and monitoring/audits of IPC practices and feedback [baseline (40), final (82.5)] recording the highest improvements. Healthcare-associated infection [baseline (10), final (25)], and built environment, materials, and equipment for IPC [baseline (43.5), final (55)] had the least improvement. Poor motivation to adopt recommended changes among hospital staff were major issues preventing improvements.
\end{abstract}

Conclusion: Promotion of IPC program and activities should be implemented at the Federal Medical Centre, Owo.

Keywords: Infection Prevention and Control, Lassa Fever, Control Assessment Framework, IPC programs, Nigeria

\section{Background}

Lassa fever is a type of viral hemorrhagic fever caused by the Lassa virus, an Arenavirus family member. Lassa fever is also known as Lassa hemorrhagic fever (LHF) [1]. LHF was named after the Lassa community in Borno State Nigeria, where it was first reported in the 1950s following two nurses' deaths from an unusual febrile illness [2,3]. Since then, the disease has affected many countries, predominantly the West Africa Region, where it is considered endemic, particularly in Benin, Ghana, Guinea, Liberia, Mali, Sierra Leone, Togo, and Nigeria [1,2].

*Correspondence: ileolasteve@yahoo.co.uk

${ }^{1}$ Department of Community Medicine, Lecturer 1, University of Ibadan, Ibadan, Oyo State, Nigeria; Department of Community Medicine, Honorary Consultant, University College Hospital, Ibadan, Oyo State, Nigeria

Full list of author information is available at the end of the article
LHF is considered an epidemic-prone disease in Nigeria, which occurs mostly during the dry season. In recent years, the disease has been trending towards endemicity in the country, with repeated outbreaks and cases being recorded during the rainy season [4]. Many LHF-infected persons are asymptomatic; however, they include headache, fever, muscle pains, backache, and vomiting during events of manifestation of symptoms. A few other periods, oral or gastrointestinal bleeding may occur. The likelihood of death occurs among one percent of LHFinfected persons and often occurs after two weeks of manifestation of the symptoms [1]. Among LHF cases that survive, hearing loss occurs among a quarter of persons and eventually resolves among half of the individuals with a hearing impairment presentation [5].

The primary mode of transmission of LHF among humans is through contact with droppings (feces or urine) of an infected 
multimammate rat. Secondary transmission from person to person is also common - thus making it a potential nosocomial infection. In fact, there have been reports of cases, including deaths among health care workers. Infection prevention and control (IPC) measures have been shown to limit spread in the hospital setting [6]. Unfortunately, knowledge and adherence to IPC measures have been sub-optimal among health care workers.[7] In early 2018, there was an unusually intense outbreak in Nigeria, which resulted in morbidity and mortality among health workers. Implementation of infection prevention and control (IPC) programs is important in controlling the LHF outbreak. We reported IPC's status at a tertiary health facility in southwest Nigeria before and after the implementation of the IPC program during a surge in the LHF outbreak.

\section{Methods}

\section{Study area}

The study was conducted at the Federal Medical Centre (FMC) located in Owo, Ondo State, Nigeria. FMC Owo is a tertiary health facility established to serve the specialized health needs of Owo, Akure, and their environs. The Emergency Operations Centre (EOC) serves as the command center for all activities during an outbreak. The various pillars of the EOC form the foundation on which the EOC functions. In the 2019 outbreak, the National Lassa fever EOC operates on six major pillars as activated by the Nigeria Centre for Disease Control: coordination, surveillance/epidemiology; case management; infection prevention, and control/safe burial; risk communication; logistics; and supplies and laboratory. Response activities revolve around these pillars.

\section{Study design}

Thispre-post quasi-experimental study was conducted to evaluate the impact of IPC training for health care workers.

\section{Study Population}

Five health care professionals responsible for organizing and implementing IPC activities at the Federal Medical Centre, Owo, who have an in-depth understanding and knowledge of IPC activities at the facility, were interviewed. The five team members used the Infection Prevention and Control Assessment Framework (IPCAF) tool in joint evaluations. The five respondents were made up of two female nurses and three male doctors. The inclusion criterion was working in the health facility for at least five years.

\section{Setting}

The study involved 3 phases: baseline assessment using the IPCAF, intervention phase, and evaluation phase. IPCAF is a close-formatted and structured questionnaire to which a scoring system has been assigned. The IPCAF has been structured in tandem with the World Health Organization (WHO) guidelines regarding the core components of IPC programs at the health facility. The IPCAF has eight divisions, which highlights the WHO IPC core components. Eighty-one indicators have been developed to measure the outcome of the implemented framework. These indicators have been developed as questions and are intended to provide an objective assessment based on the overall performance recorded in all the eight sections. The FMC, Owo, presently has assigned to her one of the four levels of IPC promotion and practice [8]. We interviewed relevant unit heads and IPC committee members.

During the second phase, we designed and implemented IPC programs based on gaps identified from the baseline assessment (see box 1). The third phase included a repeat of the assessment conducted during phase one.

Box 1 Summary of intervention delivered

- Facility dialogue

- Supportive supervision and mentorship vis a vis adherence to IPC guidelines and appropriate waste management, PPE use, workplace hygiene, including injection safety

- Training of health workers

- Provision of alcohol-based hand sanitizers, scrubs, and chlorinated water

- Sensitization on IPC, distribution of poster and handbills

Assessment tool and scoring

The tool has eight core components: IPC program, IPC guidelines; IPC education and training; Healthcare-associated infection (HAI) surveillance, multimodal strategies for implementation of IPC interventions, monitors, and audits IPC practices and feedback; and is concerned with the workload, staffing, and bed occupancy. In addition, the IPCAF is responsible for the built environment as well as the materials and equipment for IPC at the facility level. Points are allocated to individual questions depending on their importance in the context of the component being assessed. The overall score for all components is 800 . The overall score obtained across the eight subsections is therefore used to assign the health facility to one of the four levels of IPC promotion and practice [8]:

- Inadequate (scores 0-200) implies that IPC core components implementation is deficient. Significant improvement is required.

- Basic (scores 201-400) means that some aspects of the IPC core components are in place but not sufficiently implemented. Further improvement is required.

- Intermediate (scores 401-600) elucidate the proper implementation of most aspects of the IPC core components. Results in this category inform on the need for an improvement in the facility's scope and quality of implementation. It also focuses on developing long-term plans for the sustenance and promotion of the existing IPC program activities.

- Advanced (601-800) explains the full implementation of the IPC core components by a health facility in tandem with the WHO's recommendations. It also highlights that such IPC programs have been fully implemented to suit the health facility's specifications.

\section{Results}

The mean age of the five health care professionals responsible for organizing and implementing IPC activities at the Federal Medical Centre, Owo, and who have in-depth understanding and knowledge of IPC activities was $50 \pm 9.6$ years. The baseline score was 318.5 , and the final score 545 . There were varying levels of improvements post-intervention. During the baseline assessment, IPC education and training had the least score and recorded the highest improvement. The least improvement was recorded in component 8: built environment, materials, and equipment for IPC at the facility level. 
Table 1 Infection prevention and control assessment framework at the facility level core component and scores at the Federal Medical Centre, Owo

\begin{tabular}{|c|c|c|c|c|}
\hline \multirow[t]{2}{*}{ Section (Core component) } & \multicolumn{4}{|c|}{ Subtotal scores } \\
\hline & $\begin{array}{l}\text { Before } \\
\text { intervention }\end{array}$ & $\begin{array}{l}\text { After } \\
\text { intervention }\end{array}$ & Differences & $\begin{array}{l}\text { Percentage } \\
\text { improvement }\end{array}$ \\
\hline IPC program & 40 & 75 & 35 & 87.5 \\
\hline IPC guidelines & 50 & 92.5 & 42.5 & 85.0 \\
\hline IPC education and training & 20 & 70 & 50 & 250.0 \\
\hline HAI surveillance & 10 & 25 & 15 & 150.0 \\
\hline Multimodal strategies & 65 & 80 & 15 & 23.1 \\
\hline Monitoring/audits of IPC practices and feedback & 40 & 82.5 & 42.5 & 106.3 \\
\hline Workload, staffing, and bed occupancy & 50 & 65 & 15 & 30.0 \\
\hline Built environment, materials, and equipment for IPC at the facility level & 43.5 & 55 & 11.5 & 87.5 \\
\hline \multirow[t]{2}{*}{ Final total score } & 318.5 & 545 & 226.5 & \\
\hline & Basic & Intermediate & & \\
\hline
\end{tabular}

\section{Discussion}

Given that IPC training and education were one of the components with the least scores, this finding underscores the need for regular training of health care workers on IPC measures before and during epidemics. It is possible to make assumptions that health care workers are familiar with infection prevention measures; our finding, however, points out that such assumptions may be wrong. Thus, health managers and relevant bodies need to consider IPC training for health workers as urgent steps during an epidemic response. Also, it reveals the need to institute mechanisms for the monitoring and supportive supervision of IPC activities during outbreaks of infections.

We found that surveillance on HAI was sub-optimal before and after the intervention. This is not surprising, as the surveillance system requires a systemic approach with commitment and coordination from national and regional governments [9]. However, commitment and coordination are frequently suboptimal. A previous study on IPC found a similar finding whereby little improvement was observed in structures or domains that require government efforts [10].

In our survey, the respondent identified poor motivation and excess workload as barriers to effective IPC practice. These have previously been reported in another study on guidelines implementation in Kenya [11]. Heavy workload has similarly been identified as barriers to hand hygiene practice among health care workers in sub-Saharan Africa [12]. This is a critical area that has not gained much attention in service delivery in Nigeria. However, another study conducted at health facilities reported a high disregard for physicians' IPC policies, contributing to increased HAI [13]. Therefore, the findings from this study highlight the need for a reduction of workload among health care workers to bearable limits to enhance adherence to IPC measures.

\section{Study limitations}

The study was conducted over a short period in a single health facility, which could have limited the generalizability of the findings from this study. Besides, this study did not assess longterm changes in IPC practice at the selected facility. Despite these limitations, this study presented valid results regarding the differences in IPC practice at a Lassa fever treatment center before and after implementing an intensive IPC program.

\section{Conclusion}

Adherence to IPC measures is required in the control of LHF infection. Regular IPC training of healthcare workers is highly required in ensuring appropriate management of LHF infection. We hereby recommend the establishment of local teams to coordinate training and provide motivation for IPC adherence. We also recommend strengthening the surveillance system and coordinating mechanisms to ensure the coping capability of the health systems in dealing with the extra demand associated with epidemics. Regarding policy implications, it is required that IPC planning be commenced at the early stage of infectious disease outbreaks to enable the successful implementation of IPC programs.

\section{Abbreviation}

IPC: Infection Prevention and Control; LF: Lassa Fever; IPCAF: Infection Prevention and Control Assessment Framework; LHF: Lassa Hemorrhagic Fever; FMC: Federal Medical Centre; EOC: Emergency Operations Centre; WHO: World Health Organization; HAI: Healthcare-Associated Infection

\section{Acknowledgment}

We thank the management and staff of FMC Owo for their cooperation and support during the conduct of this study.

\section{Funding}

The authors received no financial support for the research, authorship, and/or publication of this article.

Availability of data and materials

Data will be available by the corresponding author at ileolasteve@yahoo.co.uk.

\section{Authors' contributions}

Olayinka Stephen Ilesanmi (OSI) is the principal investigator of this manuscript. All authors are equally participated in the study concept, design, writing, reviewing, editing, and approving the manuscript in its final form. All authors have read and approved the final manuscript.

\section{Ethics approval and consent to participate}

We conducted the research following the Declaration of Helsinki, and the Ethics Committee approved the protocol of the Federal Medical 
Centre, Owo, Ondo State, Nigeria (Ref: FMC/OW/380/VOL. LXVII/187 FMC OWO).

\section{Consent for publication}

Not applicable

\section{Competing interest}

The authors declare that they have no competing interests.

\section{Open Access}

This article is distributed under the terms of the Creative Commons $\begin{array}{llll}\text { Attribution } & 4.0 & \text { International License }\end{array}$ (http://creativecommons.org/licenses/by/4.0/), which permits unrestricted use, distribution, and reproduction in any medium, provided you give appropriate credit to the original author(s) and the source, provide a link to the Creative Commons license, and indicate if changes were made. The Creative Commons Public Domain Dedication waiver (http://creativecommons.org/publicdomain/zero/1.0/) applies to the data made available in this article, unless otherwise stated.

\section{Author details}

${ }^{1}$ Department of Community Medicine, Lecturer 1, University of Ibadan, Ibadan, Oyo State, Nigeria; Department of Community Medicine, Honorary Consultant, University College Hospital, Ibadan, Oyo State, Nigeria. ${ }^{2}$ Department of Community Health, Consultant, Federal Medical Centre, Owo, Ondo State, Nigeria. ${ }^{3}$ Department of Community Medicine, Resident, University College Hospital, Ibadan, Oyo State, Nigeria. ${ }^{4}$ Department of Microbiology, Consultant, Federal Medical Centre, Owo, Ondo State, Nigeria. ${ }^{5}$ Department of Community Health, Assistant Director of Nursing Services, Federal Medical Centre, Owo, Ondo State, Nigeria. ${ }^{6}$ Department of Community Health, Resident, Federal Medical Centre, Owo, Ondo State, Nigeria. ${ }^{7}$ Department of Pharmacy, Intern, University College Hospital, Ibadan, Oyo State, Nigeria.

\section{Article Info}

Received: 02 September 2020

Accepted: 16 October 2020

Published: 21 October 2020

\section{References}

1. World Health Organization, WHO. Lassa fever. Available from: https://www.who.int/health-topics/lassa-fever/\#tab=tab_1.

[Accessed on 11 October 2020]

2. Nigeria Centre for Disease Control. Lassa fever. Available from: https://ncdc.gov.ng/diseases/factsheet/47. [Accessed on 11 October 2020].

3. World Health Organization, WHO. Lassa fever. Available from: https://www.who.int/news-room/fact-sheets/detail/lassa-fever. [Accessed on 11 October 2020].

4. Ijarotimi IT, Ilesanmi OS, Aderinwale A, Abiodun-Adewusi O, Okon IM. Knowledge of Lassa fever and use of infection prevention and control facilities among health care workers during Lassa fever outbreak in Ondo state, Nigeria. Pan Afr Med J. 2018; 30:1-13. https://doi.org/10.11604/pamj.2018.30.56.13125

5. Mateer EJ, Huang C, Shehu NY, Paessler S. Lassa fever-induced sensorineural hearing loss: A neglected public health and social burden. PLoS Negl Trop Dis. 2018;12(2):1-11. https://doi.org/10.1371/journal.pntd.0006187

6. Ijarotimi I., Oladejo J., Nasidi A, Jegede O. Lassa fever in the State Specialist Hospital Akure, Nigeria: Case report, Contact tracing and outcome of hospital contacts. Int J Infect Trop Dis. 2016;3(1):20-28. https://doi.org/10.14194/ijitd.3.1.4

7. Ireye F, Ejiyere H, Aigbiremolen AO, Famiyesin OE, RowlandUdoh EA, Ogeyemhe CO, Okudo I, Onimisi AB. Knowledge, attitude and infection prevention and control practices regarding Lassa fever among healthcare workers in Edo State, Nigeria. Int $\mathbf{J}$ Prev Treat. 2019;8(1):21-27. https://doi.org/10.5923/j.ijpt.20190801.03

8. World Health Organization. Infection prevention and control assessment framework at the facility level. 2018; 2016:1-15. Available from: https://www.who.int/infectionprevention/tools/core-components/IPCAF-facility.PDF?ua=1 [Accessed on 11 October 2020].

9. World Health Organization, WHO. Communicable disease surveillance and response systems - Guide to monitoring and evaluating. Epidemic and pandemic alert and response. Published online 2006:90. doi: rr5305a1 [pii]

10. Ousman K, Kabego L, Talisuna A, Diaz J, Mbuyi J, Houndjo B, et al. The impact of Infection Prevention and control (IPC) bundle implementation on IPC compliance during the Ebola virus outbreak in Mbandaka/Democratic Republic of the Congo: A before and after design. BMJ Open. 2019;9(9):1-6. https://doi.org/10.1136/bmjopen-2019-029717

11. Nzinga $\mathrm{J}$, Mbindyo $\mathrm{P}$, Mbaabu L, Warira A, English $\mathrm{M}$. Documenting the experiences of health workers expected to implement guidelines during an intervention study in Kenyan hospitals. Implement Sci. 2009;4(1):1-9. https://doi.org/10.1186/1748-5908-4-44.

12. Ataiyero Y, Dyson J, Graham M. Barriers to hand hygiene practices among health care workers in sub-Saharan African countries: A narrative review. Am J Infect Control. 2019 May;47(5):565-573. https://doi.org/10.1016/j.ajic.2018.09.014.

13. Gilbert GL, Kerridge I. The politics and ethics of hospital infection prevention and control: a qualitative case study of senior clinicians' perceptions of professional and cultural factors that influence doctors' attitudes and practices in a large Australian hospital. BMC Health Serv Res. 2019; 19(212). https://doi.org/10.1186/s12913-019-4044-y. 